\title{
A note on co-occurrence, transitional probability, and causal inference
}

\author{
Jeroen van Paridon \\ MPI for Psycholinguistics \\ jeroen.vanparidon@mpi.nl
}

\author{
Phillip M. Alday \\ MPI for Psycholinguistics \\ phillip.alday@mpi.nl
}

July 2020

\begin{abstract}
Much has been written about the role of prediction in cognition in general, and language processing in particular, with some authors even claiming that prediction is the central goal of cognition. Attributing such a specific goal to cognition seems speculative, but prediction is generally held to play an important role in both perception and action. In empirical studies of language processing, however, measures of predictability such as forward transitional probability (or surprisal) are often no more effective in describing behavioral and neural phenomena than measures of post- or retrodictability such as backward transitional probability. We address this paradox by looking at the relationship between these different information theoretic measures and proposing a mechanistic account of how they are used in cognition. We posit that backward transitional probabilities support causal inferences about the occurrence of word sequences. Using Bayes' Theorem, we demonstrate that predictions (formalized as forward transitional probabilities) can be used in conjunction with the marginal probabilities of the current state/word and the upcoming state/word to compute these causal inferences. This conceptualization of causal inference in language processing both accounts for the role of prediction, and the surprising effectiveness of backwards transitional probability as a predictor of human behavior and its neural correlates.
\end{abstract}

\section{On $n$-gram frequency and conditional probability}

For at least half a century, it has been recognized high frequency ${ }^{1}$ words are easier to produce (Jescheniak \& Levelt, 1994; Oldfield \& Wingfield, 1965) and to recognize, both in speech (Broadbent, 1967; Cleland et al., 2006; Dahan et al., 2001) and in print (Cleland et al., 2006; Kuperman, 2013; Rayner, 1998). However, when modeling language

\footnotetext{
${ }^{1}$ We use the term frequency with regards to word occurrence in this article, which generally denotes a rate of occurrence (e.g., number of word occurrences per 1 million words, a scale from 0 to 1 million). Note however that for the purpose of comparing relative rates of occurrence, frequency is completely interchangeable with absolute counts (a scale from 0 to whatever the size of the corpus) and probabilities (a scale from 0 to 1 ).
} 
processing (be it speech perception, reading, etc.), we are often interested in processing beyond the single word level. Processing at the level of multi-word phrases (word $n$-grams) is more complex to model than single-word processing. This is partly due to the (linear) increase in lexical factors when modeling multi-word phrases, but more importantly, our understanding of phrase processing is not as well developed as our understanding of single-word processing.

One easily accessible statistic relevant to phrase processing is word $n$-gram frequency, which has indeed been demonstrated to affect both language comprehension (Arnon \& Snider, 2010) and language production (Janssen \& Barber, 2012; Shao et al., 2019). These $n$-gram effects occur in addition to, and are distinct from, the effect of single-word frequency (Jacobs et al., 2016; Shao et al., 2019). It has been suggested that these $n$-grams are stored as single units (lexical bundles, see e.g., Jacobs et al., 2016; Tremblay et al., 2011). However, given the combinatorial explosion of word $n$-grams that occurs for any value of $n$ greater than 1 , it is clear that storing $n$-grams (in some sort of expanded mental lexicon) is infeasible for all but the highest frequency $n$-grams (Baayen et al., 2013, Onnis \& Huettig, in prep.), making whole $n$-gram storage inconsistent with the observation that $n$-gram frequency effects affect both high and low frequency $n$-grams (Arnon \& Snider, 2010).

We therefore reject the notion that apparent $n$-gram frequency effects are caused by the storage of whole $n$-grams and their frequencies, except for phrases with frequencies high enough to classify them as idioms (or compounds, cf. Jacobs \& Dell, 2014), rather than phrases with purely compositional meaning. A more feasible mechanism than storing whole $n$-grams is to make use of conditional probabilities: The probability of a word occurring, given the occurrence of the preceding word. These conditional probabilities can be computed bidirectionally and are generally called transitional probabilities in the context of language (but note that these concepts are fundamentally equivalent). In studies of reading, the forward transional probability is generally referred to as predictability, which has been found to have a robust effects on various reading-related measures (e.g., first fixation duration, Balota et al., 1985; and inspection probability, Kliegl et al., 2004; for an alternative implementation of predictability see McDonald and Shillcock, 2003). Transitional probabilities can also be reframed as surprisal ( $\left.-\log P_{\text {conditional }}\right)$, an information theoretic measure that is often used in the field of Natural Language Processing. If we conceptualize the mental lexicon as a network of nodes and edges, transitional probabilities could feasibly be encoded in the edge weights, whereas storing whole $n$-grams requires an exponential increase in the number of nodes (cf. Baayen et al., 2013). 


\subsection{Hard to tell the difference: Equivalences}

In the following sections, we transform all relevant quantities to a logarithmic scale (which is common practice) for reasons of computational convenience and cognitive plausibility $^{2}$.

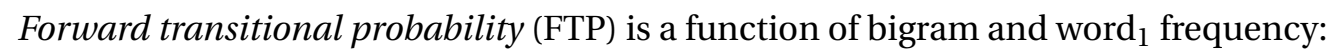

$$
\log P\left(w \mid w_{\text {prev }}\right)=\log \frac{P\left(w_{\text {prev }}, w\right)}{P\left(w_{\text {prev }}\right)}=\log P\left(w_{\text {prev }}, w\right)-\log P\left(w_{\text {prev }}\right)
$$

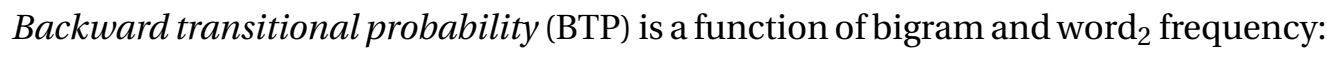

$$
\log P\left(w_{\text {prev }} \mid w\right)=\log \frac{P\left(w_{\text {prev }}, w\right)}{P(w)}=\log P\left(w_{\text {prev }}, w\right)-\log P(w)
$$

When we compile bigram occurrences from a large corpus of transcribed pseudoconversational speech (for corpus details see van Paridon \& Thompson, in press), we find a strong negative correlation between first- and second-word frequency (see Figure 1), as well as FTP and BTP (see Figure 2). Consequently, when first- and secondword frequency or FTP and BTP are both included in a linear model, the magnitude and direction of their effects will interact and therefore not be easily interpretable (if interpretable at all).

Unfortunately, things only get more confusable (and confusing) from here. Using Bayes' Theorem ${ }^{3}$ we can compute FTP from BTP (and vice versa):

$$
\log P\left(w \mid w_{\text {prev }}\right)=\log \frac{P\left(w_{\text {prev }} \mid w\right) \cdot P(w)}{P\left(w_{\text {prev }}\right)}=\log P\left(w_{\text {prev }} \mid w\right)+\log P(w)-\log P\left(w_{\text {prev }}\right)
$$

Ergo, information-theoretic surprisal, which is equivalent to negative log FTP can be computed from word frequencies and $\mathrm{BTP}^{4}$ :

$$
-\log P\left(w \mid w_{\text {prev }}\right)=\log P\left(w_{\text {prev }}\right)-\log P\left(w_{\text {prev }} \mid w\right)-\log P(w)
$$

\footnotetext{
${ }^{2}$ Evidence suggests that word frequencies are experienced (both consciously and subconsciously) on a logarithmic scale. Contrast angry and enraged, for instance: angry is a fairly frequent word and enraged is fairly infrequent (in fact, in our dataset, angry is 56 times more frequent than enraged), however the effect of this difference in frequency on the difference in e.g., reading times or lexical decision times will not be proportional to the frequency, but to the logarithm of the difference in frequency. Similarly, when asked for explicit ratings in the difference in word frequency between different words, people are likely to give answers proportional to the logarithm of the frequency. This is in line with other power laws in cognition and perception and the reason why common measurement scales such as decibels for sound intensity are logarithmic in the physical unit, but linear in perception. Note also that the base of the logarithm is irrelevant, in general, because every logarithms is a multiple of every other logarithm, so when rescaling predictors to their standard deviation (common practice for linear regression in cognitive science), because the standard deviation of a log-transformed predictor is proportional to the base of the logarithm, the rescaled predictor will be invariant with respect to the base of the logarithm.

${ }^{3}$ Bayes' Theorem as used here is simply the law of conditional probabilities. Its use here is not specific to Bayesian statistics.

${ }^{4}$ Similarly, pointwise mutual information (PMI) can be computed from frequencies:
}

$$
\log \frac{P\left(w_{\text {prev }}, w\right)}{P\left(w_{\text {prev }}\right) \cdot P(w)}=\log P\left(w_{\text {prev }}, w\right)-\log P(w)-\log P\left(w_{\text {prev }}\right)
$$




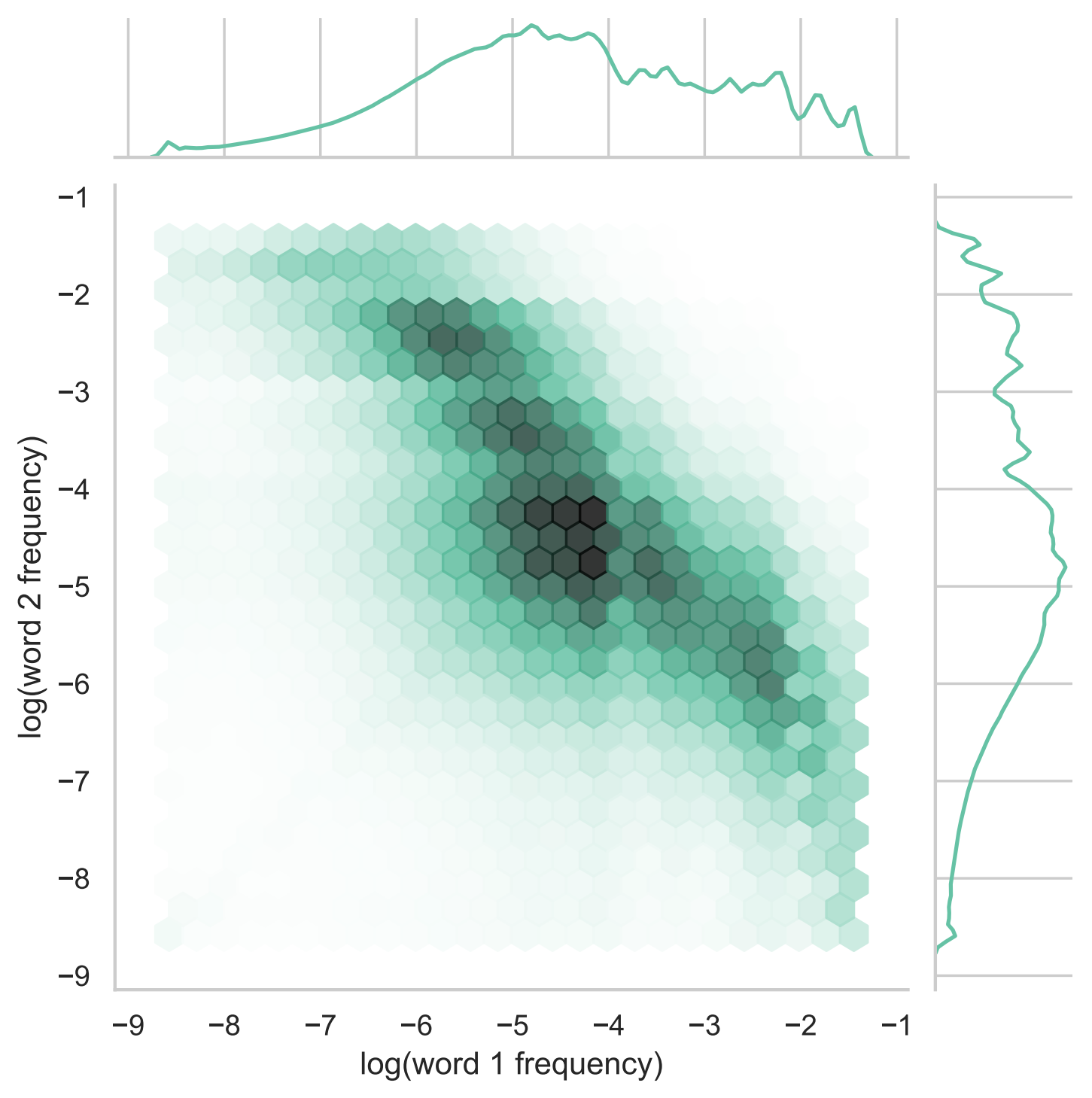

Figure 1: Joint distribution of first and last word frequencies for bigrams. 


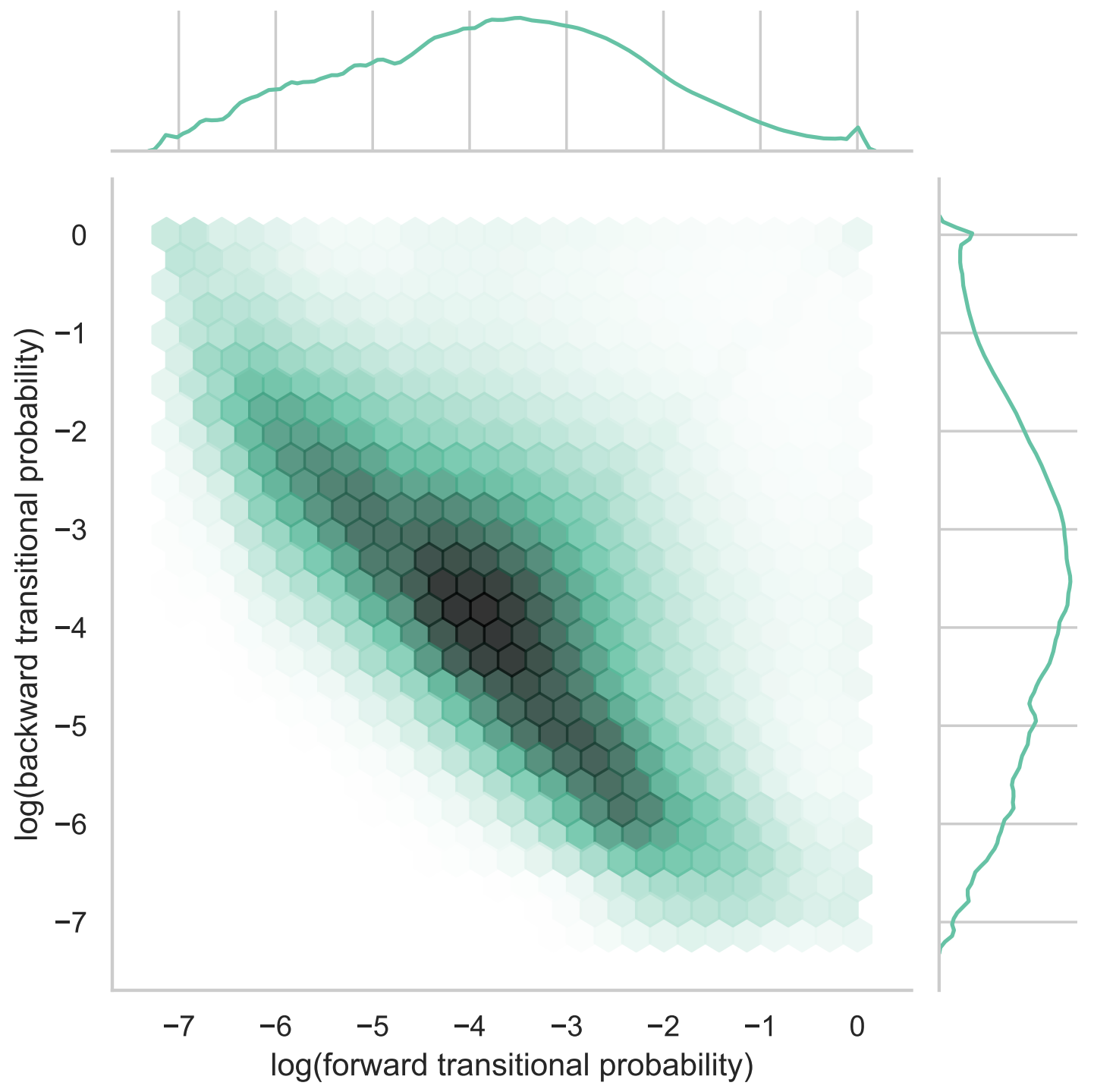

Figure 2: Joint distribution of forward and backward transitional probabilities for bigrams. 
Similar results can be derived for other information theoretic measures.

\subsection{The surprising effect of surprisal: Multicollinearity in lin- ear models of behavior}

The practical consequence of the equivalences outlined above is that when multiple measures of frequency and co-occurrence are used simultaneously as predictors in a linear model, this tends to result in collinearity between linear combinations of predictors. If this multicollinearity is perfect, it is impossible to perform the linear algebra necessary to fit the regression models. Most statistics packages will issue a warning regarding this multicollinearity and which predictors it concerns. However, even in cases where there is not perfect multicollinearity, the use of two or more co-occurrence measures can lead to unexpected consequences.

A hypothetical example: To predict reading times of a word of interest, $w$, we use $w$ frequency and FTP from $w_{\text {prev }}$ to $w$ as predictors (the former as a measure for the ease of retrieving the current word, the latter as a measure for the predictability of the upcoming word). Counterintuitively, we find that low FTP is associated with faster reading. Does this mean that surprising words are somehow also more predictable? That seems contradictory. However, let's consider a simple linear model of reaction time with word frequency and FTP as predictors. For simplicity, we leave out the error term:

$$
\log R T=\beta_{0}+\beta_{1} \cdot \log P(w)+\beta_{2} \cdot \log P\left(w \mid w_{\text {prev }}\right)
$$

Now, using Equation 3, we note that:

$$
\log P\left(w \mid w_{\text {prev }}\right)=\log P\left(w_{\text {prev }} \mid w\right)+\log P(w)-\log P\left(w_{\text {prev }}\right)
$$

And we substitute this back into the model:

$$
\log R T=\beta_{0}+\beta_{1} \cdot \log P(w)+\beta_{2} \cdot\left(\log P\left(w_{\text {prev }} \mid w\right)+\log P(w)-\log P\left(w_{\text {prev }}\right)\right)
$$

From this, it becomes clear that the apparent negative effect for FTP on actually reflects an effect of BTP minus $w_{\text {prev }}$ frequency.

$$
\log R T=\beta_{0}+\left(\beta_{1}+\beta_{2}\right) \cdot \log P(w)+\beta_{2} \cdot\left(\log P\left(w_{\text {prev }} \mid w\right)-\log P\left(w_{\text {prev }}\right)\right)
$$

Because the effect of word frequencies is so large and stable, $w_{\text {prev }}$ frequency will tend to dominate BTP and the coefficient $\beta_{2}$ will be negative. At the same time, because

And considering Equations 1 and 2, that means we can compute PMI from transitional probability (symmetrically):

$$
\log \frac{P\left(w_{\text {prev }}, w\right)}{P\left(w_{\text {prev }}\right) \cdot P(w)}=\log P\left(w \mid w_{\text {prev }}\right)-\log P(w)=\log P\left(w_{\text {prev }} \mid w\right)-\log P\left(w_{\text {prev }}\right)
$$

Many information-theoretical measures can be trivially computed from frequencies and transitional probabilities in this fashion. 
$\beta_{2}$ is negative, $\beta_{1}$ will be inflated making the effect of $w$ frequency seem larger than it is.

\section{On making theoretically motivated choices}

That certain combinations of frequency and transitional probability measures are mathematically exchangeable might seem convenient because it allows us to choose a set of predictors that is convenient for us to work with. This becomes problematic however if we try to claim that cognitive processes operate on the specific selection of quantities that we (arbitrarily) chose to model. Strong correlations and multicollinearity make it near impossible for naive statistical methods to distinguish between theoretical accounts that hinge on the primacy of one probability measure (be it transitional probability, unigram or bigram frequency, etc.) over others (see e.g., Levy, 2008, for a footnote on how FTP/BTP correlation complicates predictor selection). Fundamentally, the psychological or neurobiological implementation of processes sensitive to frequency and transitional probability matters, but we are not able to draw conclusions from these measures alone. Instead, we need to look to fundamental structural reasons why one representation would be more compatible with other structures and mechanisms, such as temporal structure, causality, and basic insights regarding neural connectivity (much the same way that arguments about frequency versus time domain representation in M/EEG are resolved by proposing fundamental mechanisms and not by computing the Fourier transform).

\subsection{Rethinking predictive coding: Retrodiction as inference}

Rather than putting prediction central in cognition, we propose that cognition functions by making probabilistic causal inferences. If we start from the assumption that at its core, cognition subserves a perception-action loop, a particularly useful cognitive mechanism would be to compute inferences about the state of the external world and the things that led to the current state (i.e., causality), as this is can guide both (imperfect) perception and subsequent action planning. Inferences about the current state of the world and the chain of states leading to the current state are encoded as backward transitional probabilities. The backward transitional probability directly answers the question "how probable is it, that the currently observed state was preceded by a given state?". This probabilistic notion of causality is the same type used in Granger causality: it does not imply causality in the philosophical or physical sense, but it does imply stochastic sequential dependence (Granger, 1969). This inference is computed via Bayes' Theorem, as above (Equation 3). In particular, we use information about marginal probabilities (of the current state (marginal likelihood) and the next state (prior)) combined with the conditional probability of the next state based on the current state (likelihood, here FTP) to compute probabilistic causality. Note that prediction occurs 
here as an intermediate step in determining causality: the likelihood, i.e., FTP, is a critical piece in computing causality. Note that this account also explains the relative success of measures such as cloze probability. In this framework, cloze probability corresponds to the maximum likelihood. In a typical experiment, where word frequencies have been carefully controlled, we thus have a manipulation of the likelihood under nearly constant priors. Because the maximum likelihood under constant priors is proportional to the maximum a posteriori value (MAP), i.e., the peak of the posterior, the standard cloze manipulation corresponds to a manipulation of the BTP. At the same time, we do not have perfect control over the prior (word frequency) in experiments, and so the maximum likelihood does not directly correspond to the MAP.

\subsection{Bayesian brains with some probabilities?}

Although we have presented our account as a direct computation of probabilities, this is not a necessity. Indeed, our account is compatible with sampling perspectives with or without direct knowledge of probabilities (cf. Sanborn \& Chater, 2016) and with variational accounts (Friston, 2005; Friston et al., 2012). It is consistent with the "reversal" of the flow of prediction and error in prominent accounts such as Friston's (2005) theory of cortical responses. In this theory, prediction flows upward through the cortical hierarchy, while error propagates downward. In our account, prediction is used to compute the probability of a given cause, which corresponds to the goodness of fit, or equivalently error, associated with that cause.

\section{Conclusion}

The rise of information theory in the brain and behavioral sciences has presented researchers with a plethora of potential quantitative measures. We find that the (arbitrary) choice of measure cannot reasonably be driven by purely statistical concerns, because commonly used measures are linear combinations of each other and thus statistically indistinguishable. This complex interrelation gives rise to apparent paradoxes, such as an illusory facilitation in processing surprising words when controlling for absolute frequency. However, these paradoxes should not be overinterpreted, as they are spurious, introduced by a particular decomposition. Instead, we should focus on computational accounts, such as the one proposed here. By assuming that inferences about causality are instrumental in both perception and action, two of the core operations of cognition, we arrive at an account of prediction as a side effect, rather than a "goal" of cognition. This account allows us to make theoretically motivated choices between information theoretic measures as predictors for language processing and human behavior more generally. 


\section{References}

Arnon, I., \& Snider, N. (2010). More than words: Frequency effects for multi-word phrases. Journal of Memory and Language, 62(1), 67-82. https:// doi.org/10.1016/j.jml. 2009.09.005

Baayen, R. H., Hendrix, P., \& Ramscar, M. (2013). Sidestepping the combinatorial explosion: An explanation of $n$-gram frequency effects based on naive discriminative learning. Language and Speech. https:// doi.org/10.1177/0023830913484896

Balota, D. A., Pollatsek, A., \& Rayner, K. (1985). The interaction of contextual constraints and parafoveal visual information in reading. Cognitive Psychology, 17(3), 364390. https:// doi.org/10.1016/0010-0285(85)90013-1

Broadbent, D. E. (1967). Word-frequency effect and response bias. Psychological Review, 74(1), 1. https://doi.org/10.1037/h0024206

Cleland, A. A., Gaskell, M. G., Quinlan, P. T., \& Tamminen, J. (2006). Frequency effects in spoken and visual word recognition: Evidence from dual-task methodologies. Journal of Experimental Psychology: Human Perception and Performance, 32(1), 104. https:// doi.org/10.1037/0096-1523.32.1.104

Dahan, D., Magnuson, J. S., \& Tanenhaus, M. K. (2001). Time Course of Frequency Effects in Spoken-Word Recognition: Evidence from Eye Movements. Cognitive Psychology, 42(4), 317-367. https://doi.org/10.1006/cogp.2001.0750

Friston, K. (2005). A theory of cortical responses. Philosophical Transactions of the Royal Society B: Biological Sciences, 360(1456), 815-836. https://doi.org/10.1098/rstb. 2005.1622

Friston, K., Thornton, C., \& Clark, A. (2012). Free-Energy Minimization and the DarkRoom Problem. Frontiers in Psychology, 3. https:// doi.org/10.3389/fpsyg.2012. 00130

Granger, C. W. J. (1969). Investigating Causal Relations by Econometric Models and Cross-spectral Methods. Econometrica, 37(3), 424-438. https: / / doi . org / 10. $2307 / 1912791$

Jacobs, C. L., \& Dell, G. S. (2014). 'hotdog', not 'hot' 'dog': The phonological planning of compound words. Language, Cognition and Neuroscience, 29(4), 512-523. https: //doi.org/10.1080/23273798.2014.892144

Jacobs, C. L., Dell, G. S., Benjamin, A. S., \& Bannard, C. (2016). Part and whole linguistic experience affect recognition memory for multiword sequences. Journal of Memory and Language, 87, 38-58. https:// doi.org/10.1016/j.jml.2015.11.001

Janssen, N., \& Barber, H. A. (2012). Phrase Frequency Effects in Language Production. PLOS ONE, 7(3), e33202. https://doi.org/10.1371/journal.pone.0033202

Jescheniak, J. D., \& Levelt, W. J. M. (1994). Word frequency effects in speech production: Retrieval of syntactic information and of phonological form. Journal of Experimental Psychology: Learning, Memory, and Cognition, 20(4), 824-843. https: //doi.org/10.1037/0278-7393.20.4.824 
Kliegl, R., Grabner, E., Rolfs, M., \& Engbert, R. (2004). Length, frequency, and predictability effects of words on eye movements in reading. European Journal of Cognitive Psychology, 16(1-2), 262-284. https://doi.org/10.1080/09541440340000213

Kuperman, V. (2013). Reassessing word frequency as a determinant of word recognition for skilled and unskilled readers. https://doi.org/10.1037/a0030859

Levy, R. (2008). Expectation-based syntactic comprehension. Cognition, 106(3), 11261177. https://doi.org/10.1016/j.cognition.2007.05.006

McDonald, S. A., \& Shillcock, R. C. (2003). Eye movements reveal the on-line computation of lexical probabilities during reading. Psychological Science, 14(6), 648652. https://doi.org/10.1046/j.0956-7976.2003.psci_1480.x

Oldfield, R. C., \& Wingfield, A. (1965). Response Latencies in Naming Objects: Quarterly Journal of Experimental Psychology. https://doi.org/10.1080/17470216508416445

Rayner, K. (1998). Eye movements in reading and information processing: 20 years of research. Psychological Bulletin, 124(3), 372. https: / / doi .org / 10.1037 / 0033 2909.124.3.372

Sanborn, A. N., \& Chater, N. (2016). Bayesian Brains without Probabilities. Trends in Cognitive Sciences, 20(12), 883-893. https:// doi.org/10.1016/j.tics.2016.10.003

Shao, Z., van Paridon, J., Poletiek, F., \& Meyer, A. S. (2019). Effects of phrase and word frequencies in noun phrase production. Journal of Experimental Psychology: Learning, Memory, and Cognition, 45(1), 147-165. https: / / doi.org / 10.1037 / xlm0000570

Tremblay, A., Derwing, B., Libben, G., \& Westbury, C. (2011). Processing Advantages of Lexical Bundles: Evidence From Self-Paced Reading and Sentence Recall Tasks. Language Learning, 61(2), 569-613. https:// doi.org/10.1111/j.1467-9922.2010. 00622.x

van Paridon, J., \& Thompson, B. (in press). Subs2vec: Word embeddings from subtitles in 55 languages. Behavior Research Methods. https: / doi.org/10.31234/osf.io/ fcrmy 